\title{
A SHARP CARATHÉODORY'S INEQUALITY ON THE RIGHT HALF PLANE
}

\author{
BÜLENT NAFI ÖRNEK
}

\begin{abstract}
In this paper, a boundary version of Carathéodory's inequality on the right half plane is investigated. Here, the function $Z(s)$, is given as $Z(s)=1+c_{1}(s-1)+c_{2}(s-1)^{2}+\ldots$ be an analytic in the right half plane with $\mathfrak{R}(s) \leqslant A(A>1)$ for $\mathfrak{R} \geqslant 0$. We derive inequalities for the modulus of $Z(s)$ function, $\left|Z^{\prime}(0)\right|$, by assuming the $Z(s)$ function is also analytic at the boundary point $s=0$ on the imaginary axis and finally, the sharpness of these inequalities is proved.
\end{abstract}

Mathematics subject classification (2010): 30C80, 32A10.

Keywords and phrases: Carathéodory's inequality, analytic function, Schwarz lemma on the boundary.

\section{REFERENCES}

[1] T. Aliyev AzeroĞLu And B. N. Örnek, A refined Schwarz inequality on the boundary, Complex Variables and Elliptic Equations 58 (2013), 571-577.

[2] H. P. BoAs, Julius and Julia: Mastering the Art of the Schwarz lemma, Amer. Math. Monthly 117 (2010), 770-785.

[3] D. M. BuRns And S. G. KRANTZ, Rigidity of holomorphic mappings and a new Schwarz Lemma at the boundary, J. Amer. Math. Soc. 7(1994), 661-676.

[4] D. Chelst, A generalized Schwarz lemma at the boundary, Proc. Amer. Math. Soc. 129 (2001), 3275-3278.

[5] V. N. Dubinin, The Schwarz inequality on the boundary for functions regular in the disc, J. Math. Sci. 122 (2004), 3623-3629.

[6] G. M. Golusin, Geometric Theory of Functions of Complex Variable [in Russian], 2nd edn., Moscow 1966.

[7] G. KRESIN AND V. MAZ'YA, Sharp real-part theorems. A unified approach., Translated from the Russian and edited by T. Shaposhnikova. Lecture Notes in Mathematics, 1903. Springer, Berlin, 2007.

[8] M. MATElJEvic, Rigidity of holomorphic mappings \& Schwarz and Jack lemma, DOI:10.13140/RG.2.2.34140.90249, In press.

[9] M. Mateljevic, Schwarz type inequalities for harmonic and related functions in the disk and the ball, IV Conference of Mathematics and Computer Science (Konferencja MatematycznoInformatyczna) Congressio-Mathematica September 20-23, 2018, at Mierki.

[10] M. Mateljevic, Schwarz lemma and distortion for harmonic functions via length and area, arXiv:1805.02979v1 [math.CV] 8 May 2018.

[11] M. Mateljevic, A. Khalfallah, Schwarz lemmas for mappings with bounded Laplacian, arXiv:1810.08823v1 [math.CV].

[12] M. MATELJEVIC AND M. SVETLIK, Hyperbolic metric on the strip and the Schwarz lemma for HQR mappings, Submitted on 20 Aug 2018, arXiv:1808.06647v1 [math.CV].

[13] P. R. MERCER, Sharpened Versions of the Schwarz Lemma, Journal of Mathematical Analysis and Applications, 205 (1997), 508-511.

[14] P. R. MERCER, Boundary Schwarz inequalities arising from Rogosinski's lemma, Journal of Classical Analysis, 12 (2018), 93-97.

[15] R. Osserman, A sharp Schwarz inequality on the boundary, Proc. Amer. Math. Soc. 128 (2000), 3513-3517. 
[16] B. N. ÖRNEK, The Carathéodory's inequality on the boundary for the holomorphic functions in the unit disc, Journal of Mathematical Physics, Analysis, Geometry 12 (2016), 287-301.

[17] B. N. ÖRneK, Carathéodory's inequality on the boundary, J. Korean Soc. Math. Ser. B: Pure Appl. Math. 22(2015), 169-178.

[18] B. N. ÖRNEK, Sharpened forms of the Schwarz lemma on the boundary, Bull. Korean Math. Soc. 50 (2013), 2053-2059.

[19] B. N. ÖRnEK AND T. DÜZENLI, Boundary Analysis for Derivative of Driving Point Impedance Functions, IEEE Transactions on Circuits and Systems II: Express Briefs. 65(9)(2018), 1149-1153.

[20] B. N. ÖRnek And T. DüZEnli, Bound Estimates for the Derivative of Driving Point Impedance Functions, Filomat. 32(18)(2018), 6211-6218. 